\title{
Gear Box Fault Diagnosis using Hilbert Transform and Study on Classification of Features by Support Vector Machine
}

\author{
Saravanan Natarajan \\ Department of Engineering, Mechanical and Industrial Engineering, Higher College \\ of Technology \\ Ministry of Manpower, Muscat, Sultanate of Oman \\ saravanan.natarajan@hct.edu.om,natarajan.saravanan1@gmail.com
}

\begin{abstract}
The condition of an inaccessible gear in an operating machine can be monitored using the vibration signal of the machine measured at some convenient location and further processed to unravel the significance of these signals. Demodulation is an important is ue in gearbox fault detection. Non-stationary modulating signals increase difficulties of demodulation. Though wavelet packet transform has better time-frequency bocalization, because of the existence of meshing frequencies, their harmonics, and coupling frequencies generated by modulation, fault detection results using wavelet packet aransform alone are usually unsatisfactory. This paper proposes a fault detection method that combines Hilbert transform and machine learning method namely support vector machines (SVMs). The statistical feature vectors from Hilbert transform coefficiens areclassified using J48 algorithm and the predominant features were fed as input for training and testing SVM and their efficiency in classifying the faults in the Bevel Gear Box was studied.
\end{abstract}

Keywords: Hilbert Transform, Support Vector Machine, Machine Learning Method, Bevel Gear Box, Statistical Features, Fault detection

\section{Introduction}

Fault diagnosis of gearboxes is of crucial importance and has been studied for several decades. A distinct characteristic of gearbox faults is that they often generate vibration signals with amplitude and/or phase modulation. In the frequency domain, this is reflected by the increase of the sideband components around the related meshing frequencies and their harmonics [1]. In general, a fault that has distributed effects, such as gear eccentricity, generates only a few sideband components. Spectral analysis is usually effective in detection of such faults. In contrast, a localized fault such as tooth crack excite transients into the background vibration, which results in a sequence of sideband components in the spectrum. In the early development stage of the localized fauls, the sideband components are not easy to recognize from the spectrum because they are distributed in broad frequency bands and may overlap with some background components.

Sideband distance may vary with time. As a result, the spectral composition of the collected vibration signal often changes with time. Fourier analysis is unable to reveal such characteristics [2]. Other approaches are needed to identify early fault features from non-stationary signals.

When gear fault occurs, the vibration signals always display non-stationary behavior. Therefore how to extract the fault characteristic information from the non-stationary 
vibration signals is the crux of the gear fault diagnosis [3]. The short-time Fourier transform is probably the most widely used time-frequency representation. However, its resolution is often unsatisfactory because of poorly matched windows in signal analysis [4]. Though Wigner-Ville distribution, another time-frequency method, is also used in fault detection [5], the oscillating interference between the signal components often exists when applied to a multi-component signal [2]. The Choi-Williams distribution provides better resolution than the smoothed Wigner-Ville distribution; however, it is still insensitive to the time-scale of signal components [4]. The smoothed instantaneous power spectrum method, which combines the advantages of the spectrogram and the instantaneous power spectrum, gives clearer time-frequency representation of signal [2]. However, weak signals may be lost in the process. However, when a signal acquired is transient in nature, spectral analysis is inherently unsuitable for detection of sidebands [6].

Wavelet transform (WT) uses a more general function as the basis on which a signal is constructed and has been used in fault detection [7] and [8]. It is actually quite suitable for analysis of non-stationary signals in gear fault detection [9]. Wavelet packets, a generalization of wavelet bases, are alternative bases formed by taking linear combinations of usual wavelet functions [10]. Wavelet packet transform (WPT) divides the frequency space into various parts and allow bet er time-requency localization of signals. It has been used as a popular diagnostic method recently [11, 12] and [13]. Nikolaou and Antoniadis [11] and Wu and Du [12] ußed energy analysis of WPT coefficients for fault detection. Yen and Lin [13] proposed a new diagnostic method that combines WPT and neural networks.

The disadvantage of WT in gearbox fault diagnosis is that all frequency components including the meshing frequency and their harmonics, as well as coupling frequencies generated by meshing frequencies, rotation requencies and noise, will all appear in the time-frequency domain. It is theh difficult to detect the fault frequency from so many frequency components by yisual inspection. More wavelet decompositions will be needed if we want to inspect the signal existing in a specific frequency band. This will require more calculation time and computer resource.

The Hilbert transform play an important role in many subjects of science and technology such as optics [14], waves in stratified fluids [15] and [16], signal processing and so on. As early as in 1940s, Gabor used Hilbert transform to define the instantaneous frequency of a non-stationary signal. In 1970s, it was proved by Vakman that under some proper physical hypothesis, the Hilbert transform is necessary for the definition of the instantaneous frequency. Not long ago, Huang developed a new method, called Hilbert-Huang transform, for analyzing nonlinear and non stationary data in $[17]$.

Suppor Vector Machine (SVM) is used in many applications of machine learning because of Its high accuracy and good generalization capabilities. SVM is based on statistical learning theory. SVM classifies better than ANN because of the principle of risk minimization. In Artificial Neural Network (ANN) traditional Empirical Risk Minimization (ERM) is used on training data set to minimize the error. Wang and McFadden [18] utilized the wavelet transform to represent all possible types of transients in vibration signals generated by faults in a gearbox. Petrille et al., [19] proposed the neural network to diagnose a simple gear system after the data have been pre-processed by the wavelet transform. Boulahbal et al., [20] used the wavelet transform to analyze the vibration signal from the gear system with pitting on the gear. 
The raw vibration signal in any mode from a single point on a machine is not a good indicator of the health or condition of a machine.

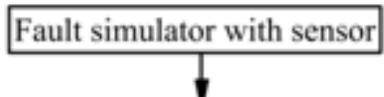

Data acquisition and

Signal conditioning

Feature extraction

Featureselection

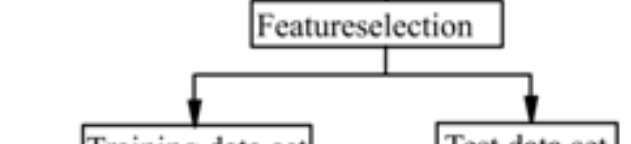

Training data set

1

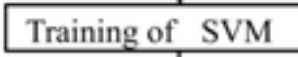

Figure 1. Flowchart of Fault Diagnosis System

Machine Condition Diagnosis

The raw ignatures acquired through a vibration sensor needed further processing and classification of the data for any meaningful surveillance of the condition of the system being monitored. The flowchart in Figure 1 explains the process of data acquisition and further processing using SYM.

This work deals with extraction of features from the vibration data of a bevel gear box system using Hilbert transform and classification of gear faults using support vector machine $(\mathrm{SVM})$. The vibration signal from a piezoelectric transducer is captured for the following conditions: Good bevel gear, bevel gear with tooth breakage (GTB), bevel gear with crack at root of the tooth (GTC), and bevel gear with face wear of the teeth (TFW) for various loading and lubrication conditions.

\section{Experimental Studies}

The fault simulator with sensor is shown in Figure 2 and the details of bevel gear box are shown in Figure 3. A variable speed DC motor $(0.5 \mathrm{hp})$ with speed up to $3000 \mathrm{rpm}$ is the basic drive. A short shaft of $30 \mathrm{~mm}$ diameter is attached to the shaft of the motor through a flexible coupling; this is to minimize effects of misalignment and transmission of vibration from motor. 


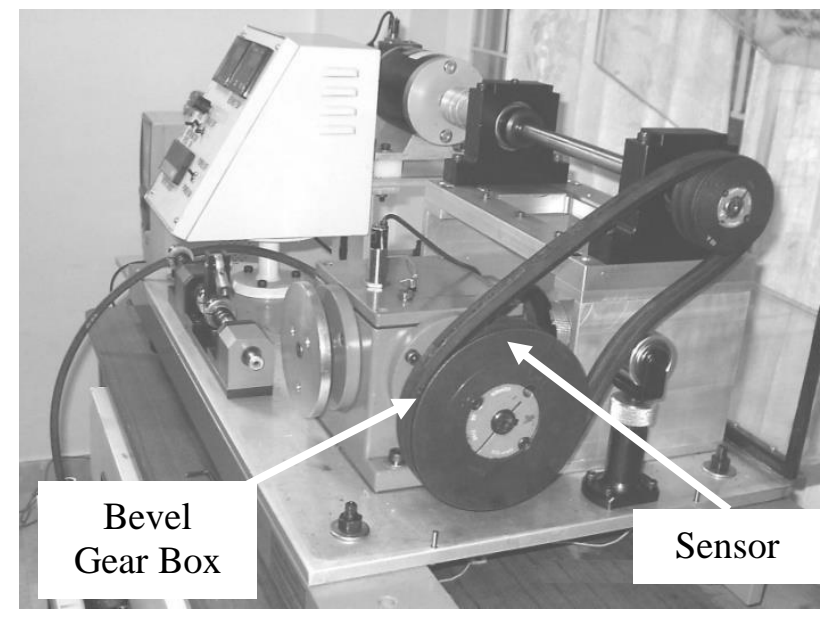

Figure 2. Fault Simulator Setup

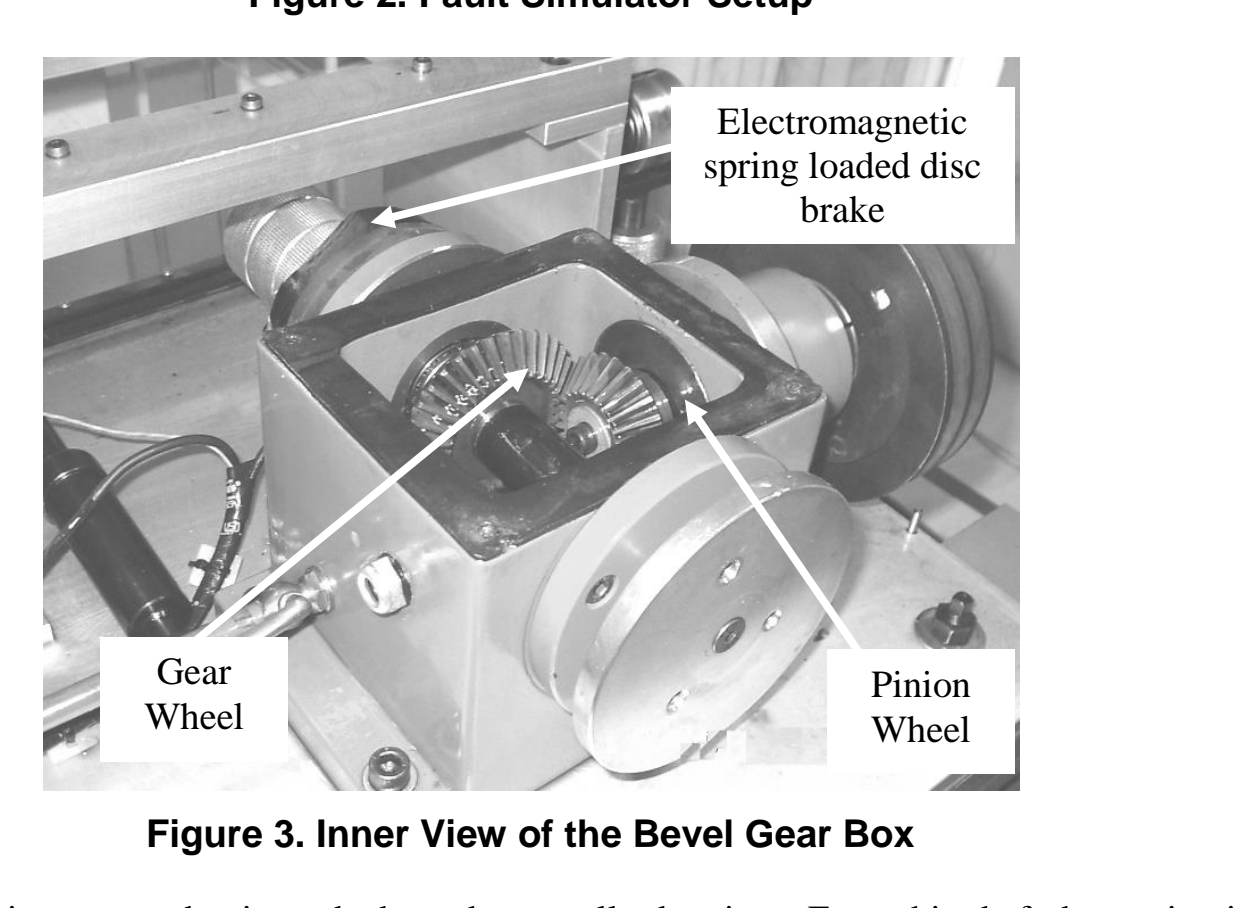

The shaft is supported at its ends through two roller bearings. From this shaft the motion is transmitted to the bevel gear box by means of a belt drive. The gear box is of dimension $150 \mathrm{~mm}$ X $170 \mathrm{~mm}$ X $120 \mathrm{~mm}$ and the full lubrication level is $110 \mathrm{~mm}$ and half lubrication level is $60 \mathrm{~mm}$ SAE 40 oil was used as a lubricant. An electromagnetic spring loaded disc brake was used to load the gear wheel. A torque level of $8 \mathrm{~N}-\mathrm{m}$ was applied at the full load condition. The various defects are created in the pinion wheels and the mating gear wheel is not disturbed. With the sensor mounted on top of the gear box vibrations signals are obtained for various conditions. The selected area is made flat and smooth to ensure effective coupling. A piezoelectric accelerometer (Dytran model) is mounted on the flat surface using direct adhesive mounting technique. The accelerometer is connected to the signal-conditioning unit (DACTRAN FFT analyzer), where the signal goes through the charge amplifier and an Analogue-to-Digital Converter (ADC). The vibration signal in digital form is fed to the computer through a USB port. The software RT Pro-series that accompanies the signal conditioning unit is used for recording the signals directly in the computer's secondary 
memory. The signal is then read from the memory and replayed and processed to extract different features.

\subsection{Experimental Procedure}

In the present study, four pinion wheels whose details are as mentioned in Table 1 were used. One was a new wheel and was assumed to be free from defects. In the other three pinion wheels, defects were created using EDM in order to keep the size of the defect under control. The details of the various defects are depicted in Table 2 and its views are shown in Figure 4.

Table 1. Details of Faults Under Investigation

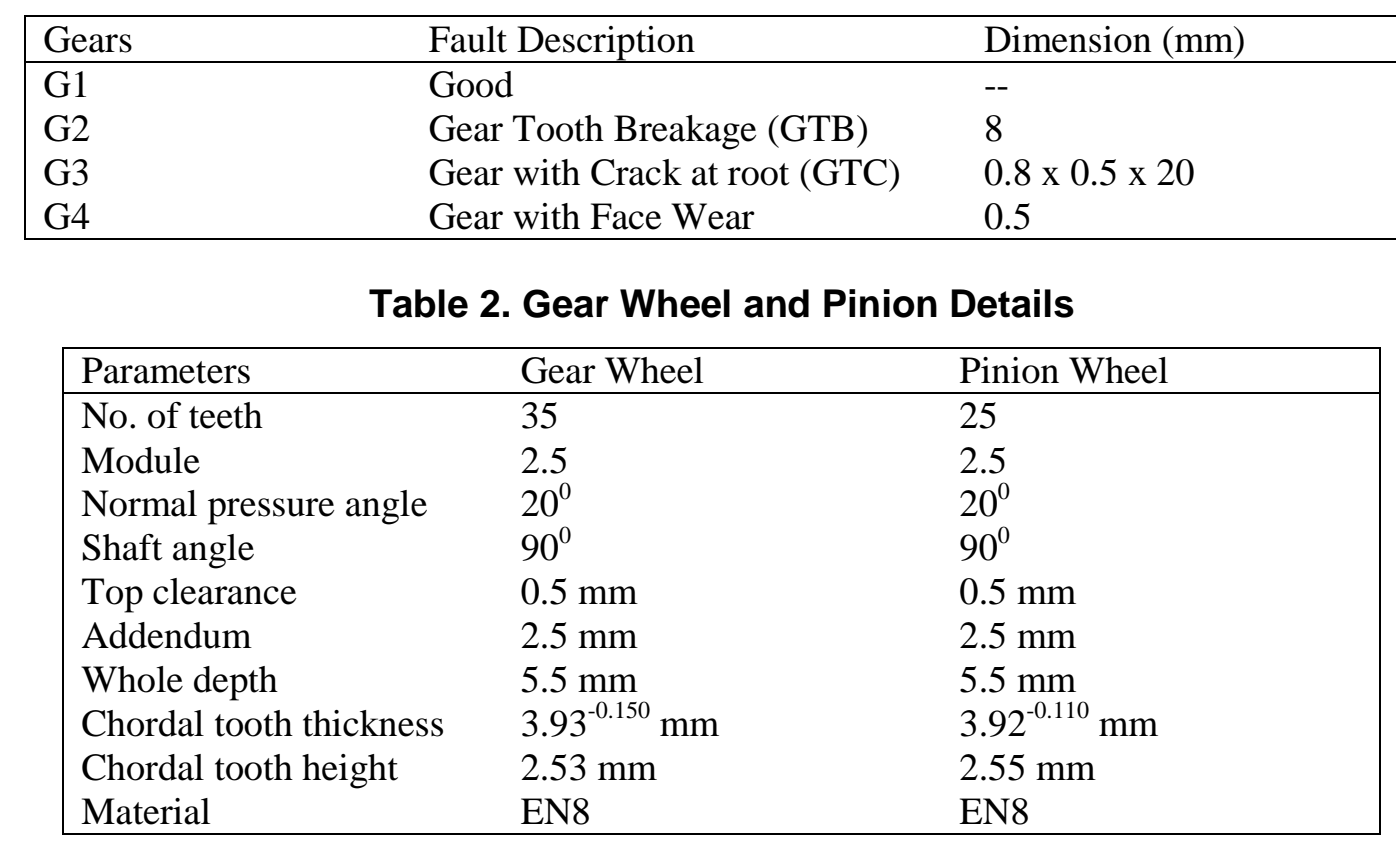

The size of the defects is a little bigger than one can encounter in the practical situation; however, it s in-line with vork reported in literature [21]. The vibration signal from the piezoelectric pickup mounted on the test bearing was taken, after allowing initial running of the gear box for some nime.

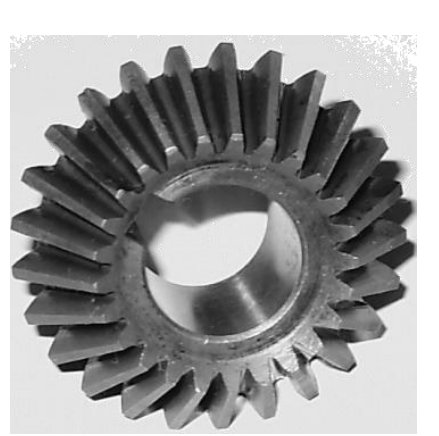

Figure 4. (a) View of Good
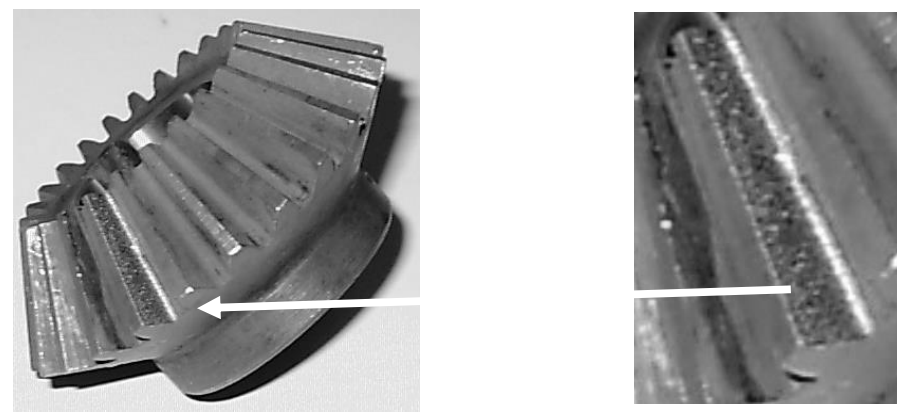

4(b) View of Pinion Wheel with Face Wear (GFW) 

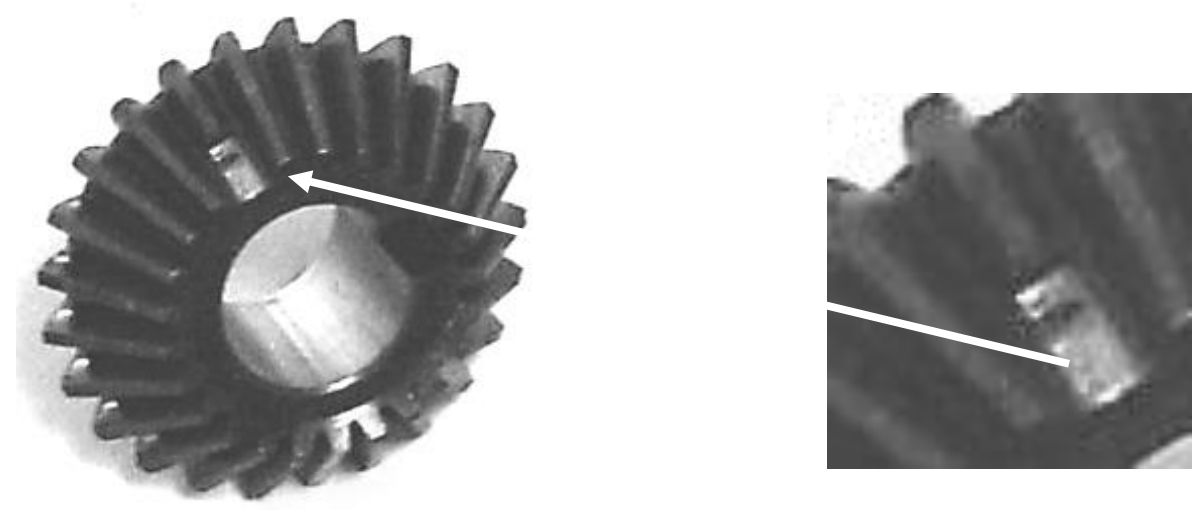

Figure 4. (c) View of Pinion Wheel with Tooth Breakage (GTB)

The sampling frequency was $12000 \mathrm{~Hz}$ and sample length was 8192 for all speeds and all conditions. The sample length was chosen arbitrarily; however, the following points were considered. Statistical measures are more meaningful, when the humber of samples is more. On the other hand, as the number of samples increases the computational time increases. To strike a balance, sample length of around 10000 was chosen. In some feature extraction techniques, which will be used with the same data, the number of samples is to be $2^{\mathrm{n}}$. The nearest $2^{\mathrm{n}}$ to 10,000 is 8192 and hence, it was taken as sample length. Many trials were taken at the set speed and vibration signal was stored in the data. The raw vibration signals acquired for various experimental conditions form the gear box using FFT are shown in Figure 5.

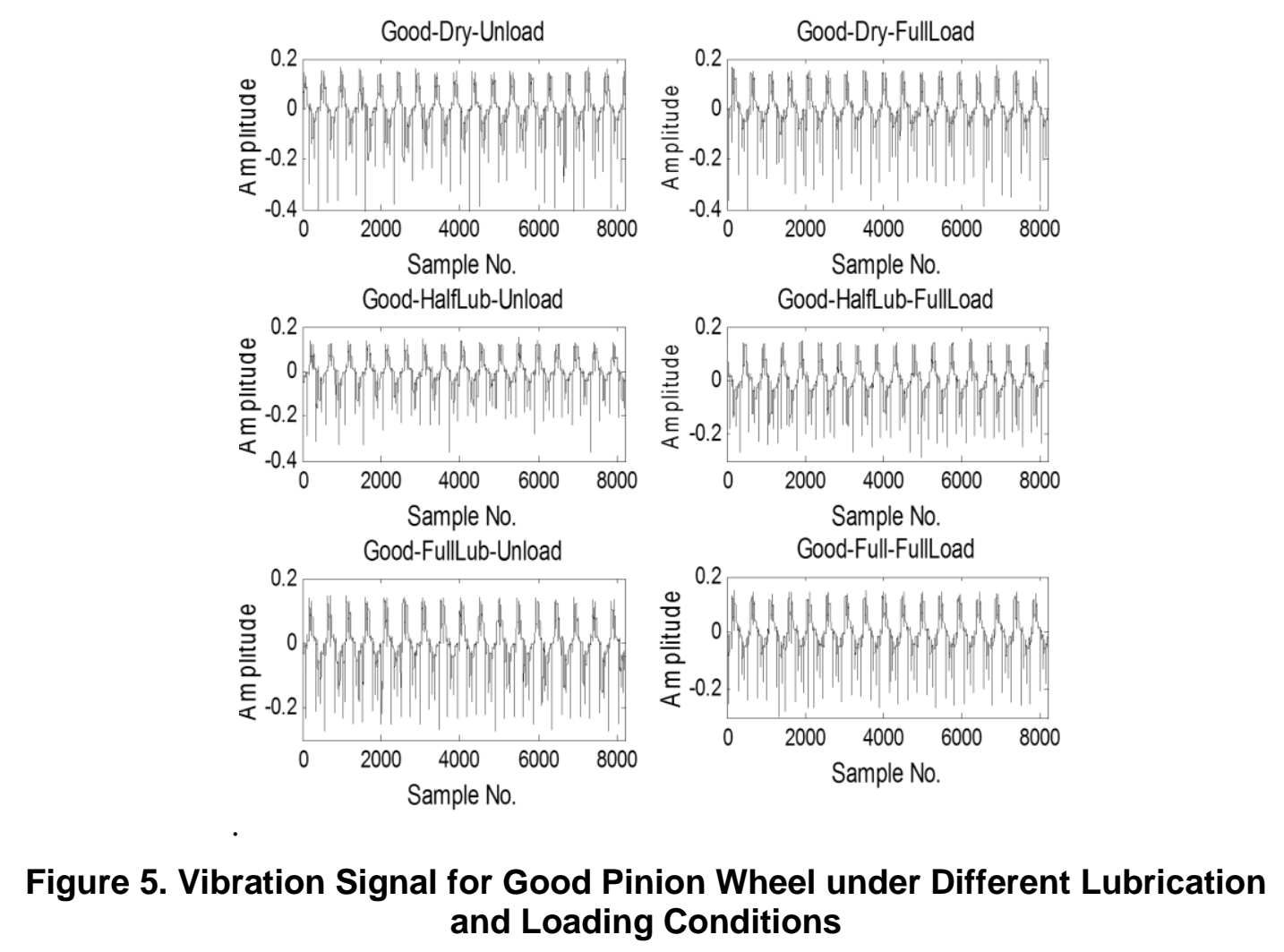




\section{Feature Extraction}

\subsection{Hilbert Transform}

Hilbert transform is a time-domain convolution that maps one real-valued time-history into another. It is defined by [22].

$H[x(t)]=\frac{1}{\pi} \int_{-\infty}^{\infty} \frac{x(t)}{t-\tau} d \tau$

where $t$ is time, $x(t)$ is a time domain signal, and $H[x(t)]$ is the Hilbert transform of $x(t)$. Because Hilbert transform is a frequency-independent $90^{\circ}$ phase shifter, the non-stationary characteristics of the modulating signal are not affected by this method at all. Amplitude and phase modulation functions, $a_{m}(t)$ and $b_{m}(t)$, respectively, can be extracted by Hilbert transform [23]. Demodulation is accomplished by forming a complex-valued time-domain signal called the analytic signal, $A[x(t)]$, which is defined as

$A[x(t)]=x(t)+i H[x(t)]=a(t) e^{i \emptyset(t)}, i=\sqrt{-1}$

The resulting complex time-domain signal can be converted from the real/1maginary format to the magnitude/phase format given below $a(t)=\sqrt{x^{2}(t)}+H^{2}[x(t)]$

$\varnothing(t)=\arctan \frac{H[x(t)]}{x(t)}$

Hilbert transform produces a complex time series. The envelope is the magnitude of this complex time series and represents an estimate of the modulation present in the signal due to sidebands [24]. It reveals modulation Insignals caused by a faulty gear. In addition, it removes carrier signals and this will decrease the onfluence of irrelevant information for the purpose of gearbox fault detection and be helpful for identification of the fault in the timefrequency plot via visual inspection. The Hilbert transform is applied to the vibrations signal of the gear box and Figure 6(a) to Figure (b), shows the Hilbert transform plots for different conditions of the gear box under study.

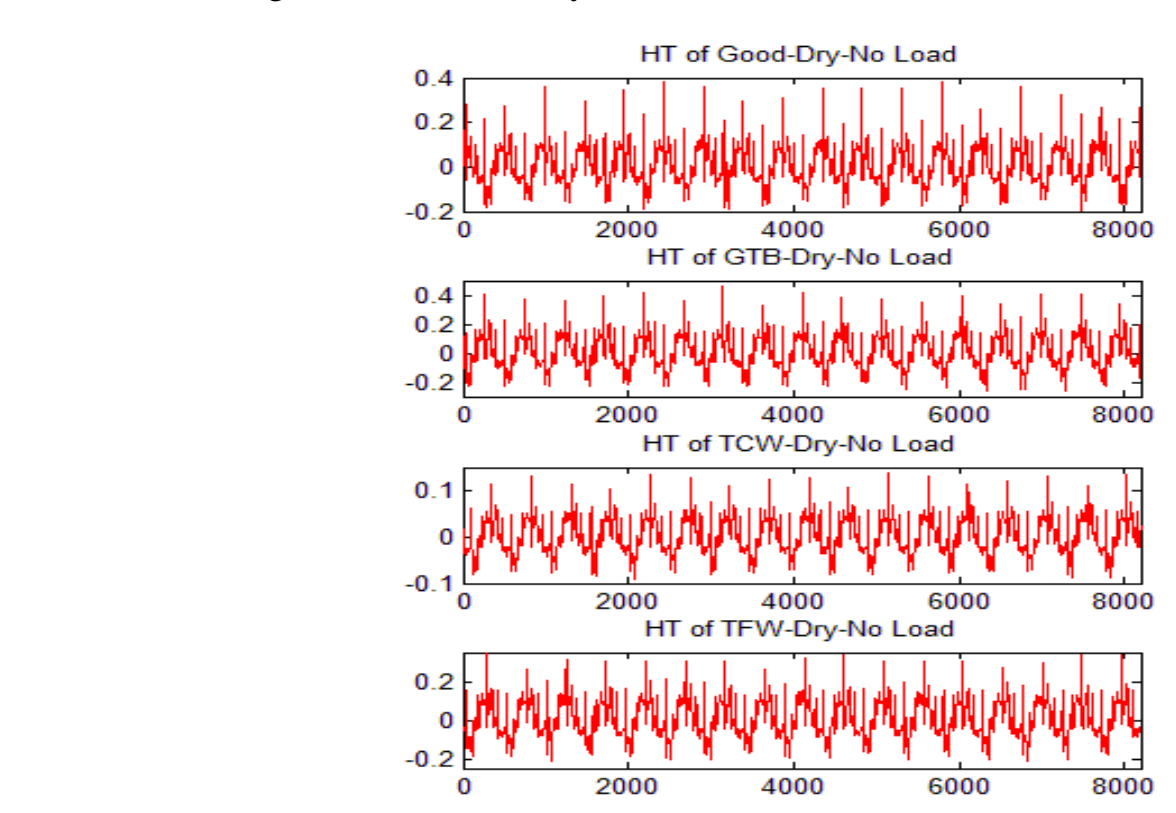

Figure 6a. Hilbert Transform Plot for Dry Lubrication and No Load Condition 
In the engineering applications, we always like to directly use some existing software packages to compute the Hilbert transform. A built-in function named 'hilbert' in Matlab, an extensively used scientific and engineering software tool, is designed for the computation. For a vector $v$ of finite data, the action of 'hilbert' on $v$ produces a complex vector Hilbert (v) of the same dimension, whose imaginary is the Hilbert transform of $v$. The principle of computing the Hilbert transform of $v$ by 'hilbert' in Matlab is to calculate the fast Fourier transform (FFT) $F v$ of $v$ firstly, multiply $F v$ by the sign multiplicator $-i \operatorname{sgn}(\cdot)$, and apply the inverse fast Fourier transform (IFFT) to get the discrete Hilbert transform of $v$. In the sequel, we call the method for the computation of the Hilbert transform with 'hilbert' in matlab the matlab method, or MM for short. Using matlab the Hilbert transform coefficients of the gear box vibration signal is extracted and the corresponding statistical features are extracted and fed as input for $\mathbf{J} 48$ algorithm for identifying the best features.

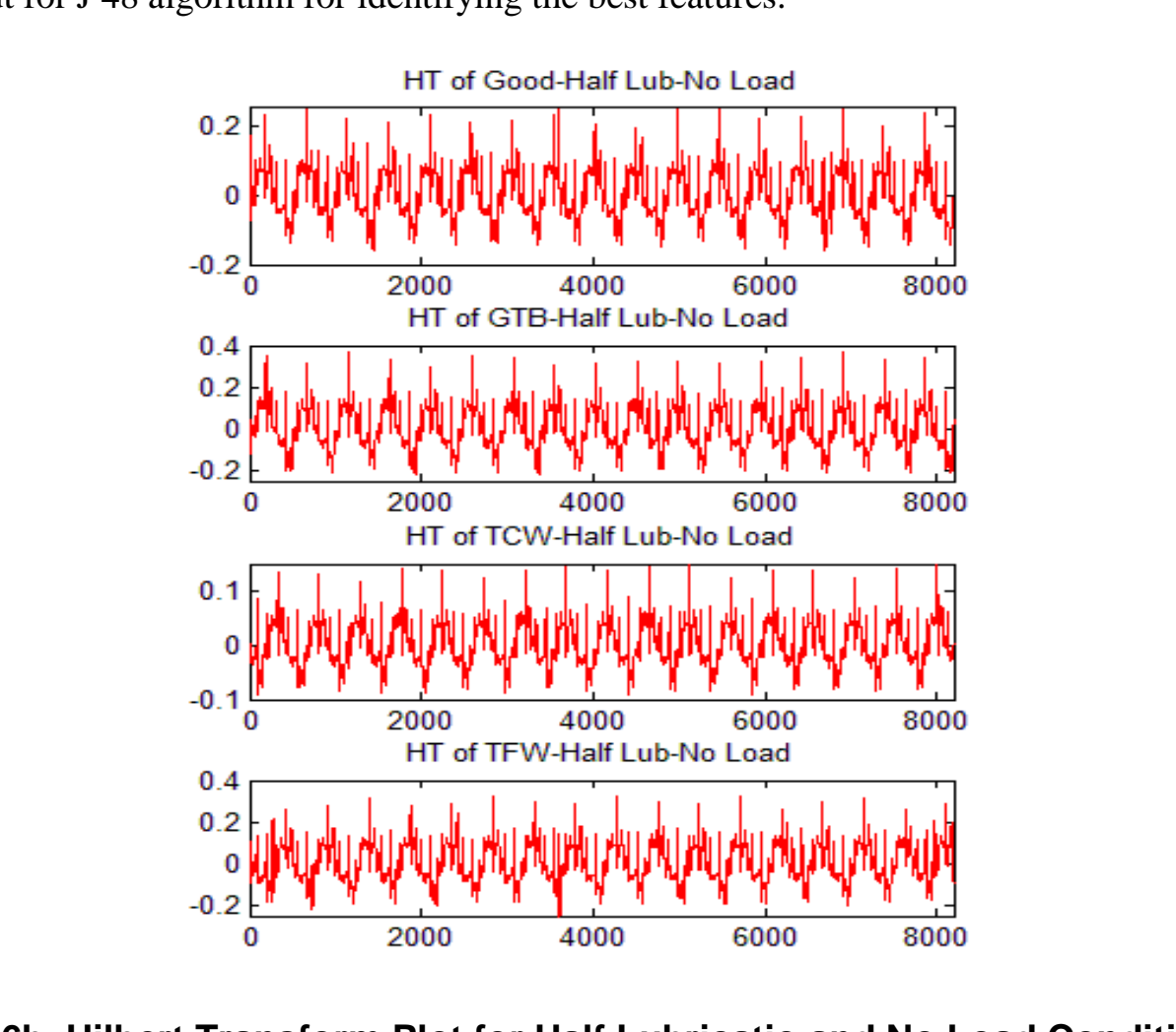

Figure 6b. Hivoert Transform Plot for Half Lubricatio and No Load Condition

\section{Using J8Algorithm in the Present Work}

A standard tree induced with c5.0 (or possibly ID3 or c4.5) consists of a number of branches, one root, a number of nodes and a number of leaves. One branch is a chain of nodes from root to a leaf; and each node involves one attribute. The occurrence of an attribute in a tree provides the information about the importance of the associated attribute as explained in [25]. A Decision Tree is a tree based knowledge representation methodology used to represent classification rules. J48 algorithm (A WEKA implementation of c4.5 Algorithm) is a widely used one to construct Decision Trees as explained in [26].

The decision tree algorithm has been applied to the problem under discussion. Input to the algorithm is set of statistical features of the Hilbert transform coefficients. It is clear that the top node is the best node for classification. The other features in the nodes of decision tree 
appear in descending order of importance. It is to be stressed here that only features that contribute to the classification appear in the decision tree and others do not. Features, which have less discriminating capability, can be consciously discarded by deciding on the threshold. This concept is made use for selecting good features.

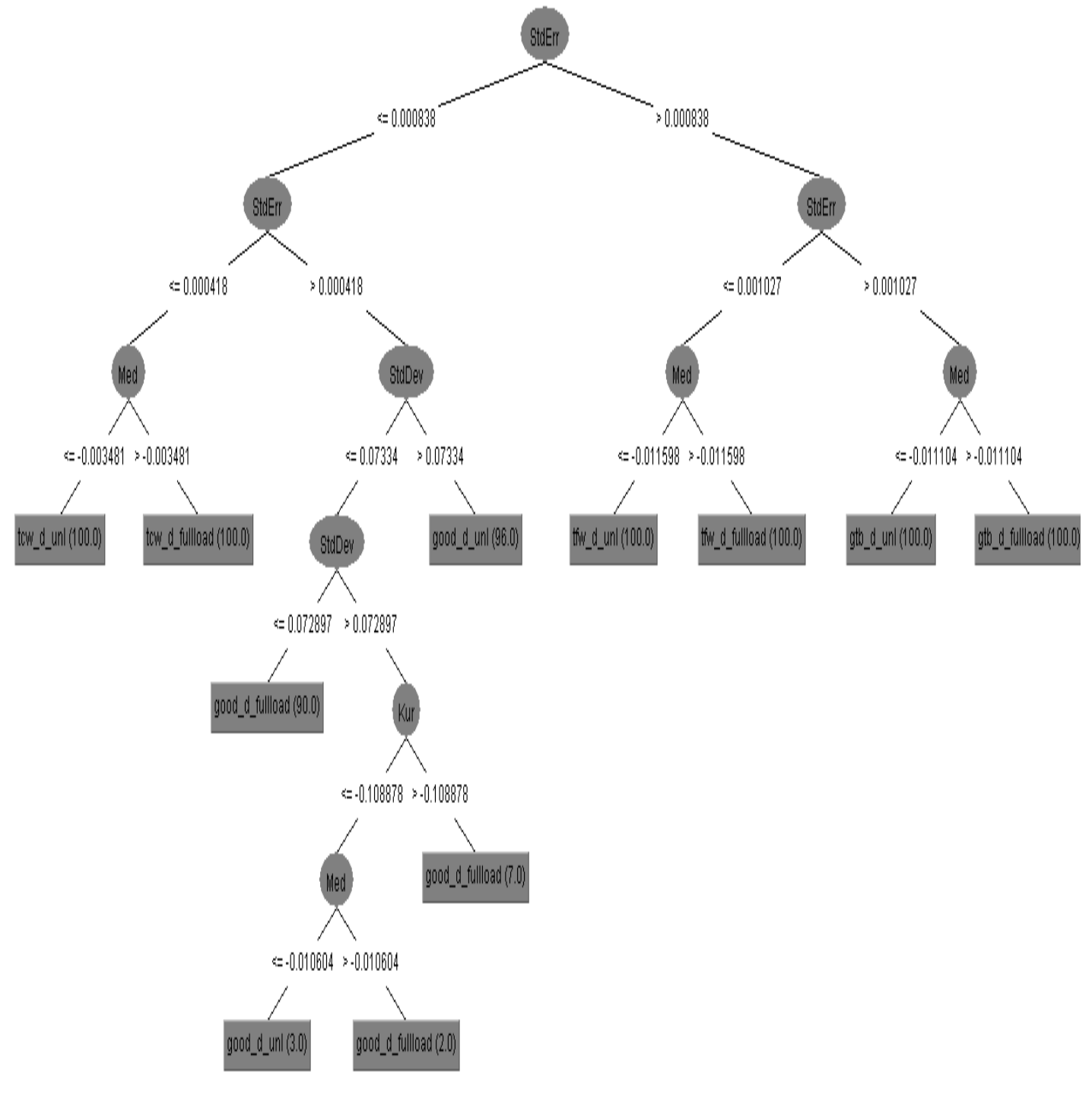

Figure 7a. Good-dry-no Load Vs GTB, GTC, TFW-dry-no Load

The algorithm identifies the good features for the purpose of classification from the given tranning data set, and thus reduces the domain knowledge required to select good features for pattern classification problem. The decision trees shown in Figure 7(a) to Figure 7(c) are output for various lubrication and loading conditions of different faults compared with good conditions of the pinion gear wheel. 


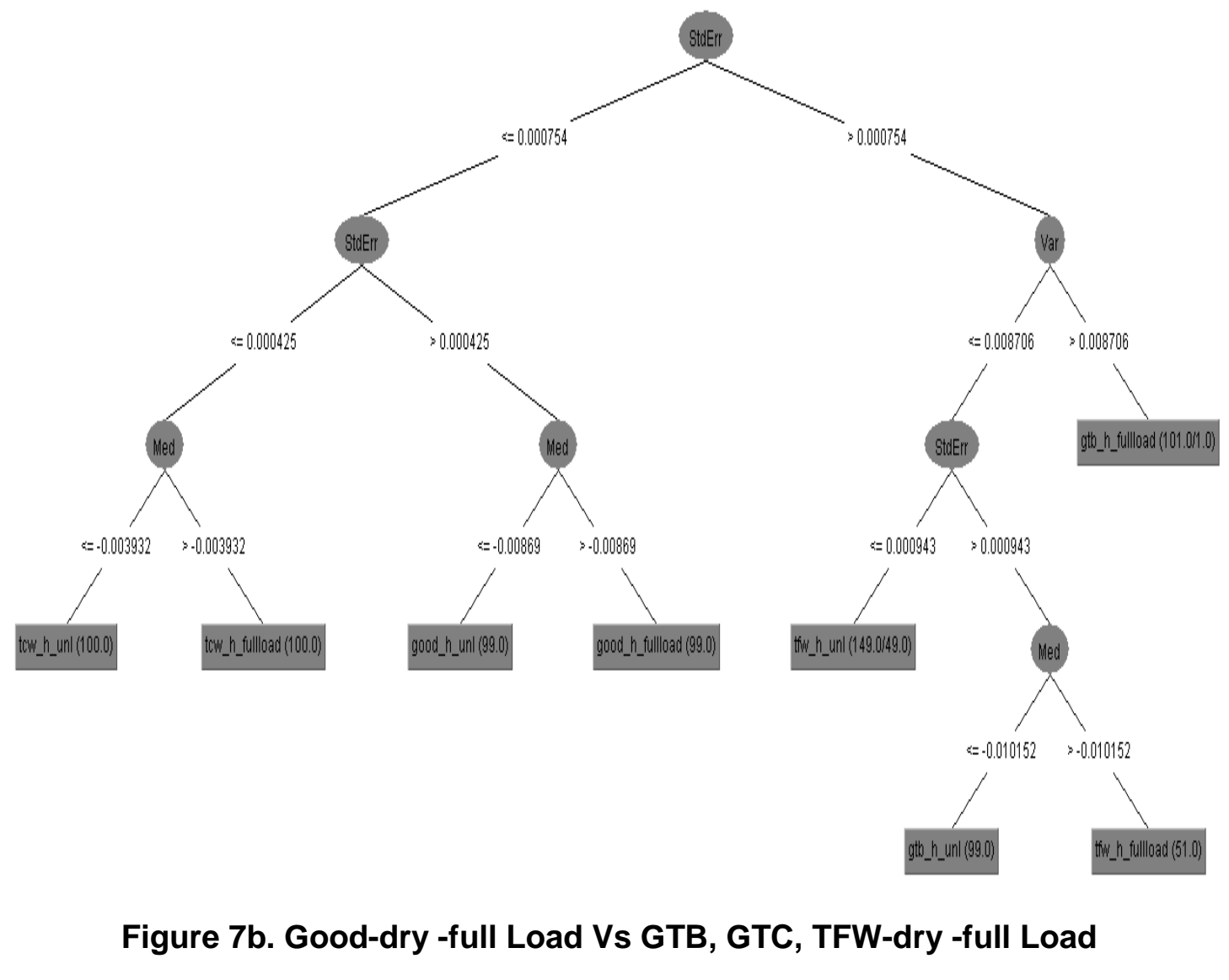

Based on above trees itsclear that of all the statistical features, standard error, median, standard deviation and ample variance play a dominant role in feature classification using Hilbert transform coefficients. These four predominant features are fed as an input to SVM for further classification.

\section{Support Vector Machine (SVM)}

The SVM is a new generation learning system based on statistical learning theory. SVM belongs to the class of supervised learning algorithms in which the learning machine is given a set of features (or inputs) with the associated labels (or output values). Each of these features can be looked upon as a dimension of a hyper-plane. SVM's construct a hyper-plane that separates the hyper-space into two classes, while doing so, SVM algorithm tries to achieve maximum separation between the classes (See Figure 8). Separating the classes with a large margin minimizes the expected generalization error. By 'minimum generalization error' we mean that when a new set of features (that is data points with unknown class values) arrive for classification, the chance of making an error in the prediction (of the class to which it belongs) based on the learned classifier (hyper-plane) should be minimum. Intuitively, such a classifier is one, which achieves maximum separation-margin between the classes. The above process of maximizing separation leads to two hyper-planes parallel to the separating plane, on either side of it. These two can have one or more points on them. The planes are known as 'bounding planes' and the distance between them is called as 'margin'. By SVM 'learning', we mean, finding a hyper-plane, which maximizes the margin and 
minimizes the misclassification error. The points lying beyond the bounding planes are called support vectors. The data points $P 1, P 2, P 3, P 4$, and $P 5$ belonging to A- are support vectors (See Figure 8), but P6, $P 7$ are not. Same facts hold good for class A+. These points play a crucial role in the theory and hence the name Support Vector Machines. Here, by 'machine', we mean an algorithm. The notations used by Fung [27] have been followed. In the formulation, ' $\mathrm{A}$ ' is a $\mathrm{m} \times \mathrm{n}$ matrix whose elements belong to real space, ' $D$ ' is $\mathrm{m} \times 1$ matrix representing class label $(+1$ and -1$)$, ' $e$ ' is a vector of ones and ' $v$ ' is a control parameter that defines the weight of error.

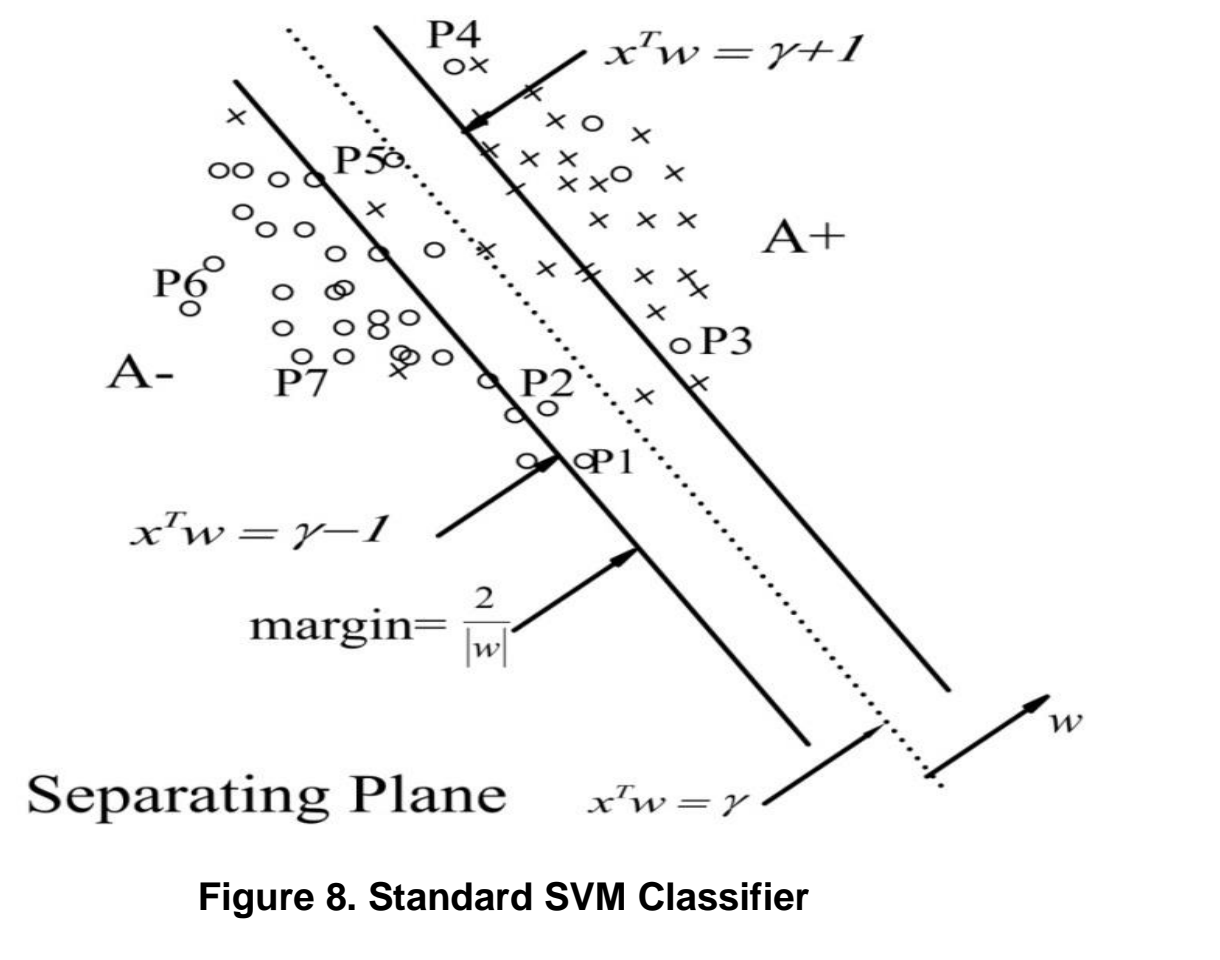

minimization and bounding plane separation in the objective function. ' $w$ ' is orientation parameter and ' $\%$ is location parameter (location relative to origin)of separating hyper plane.

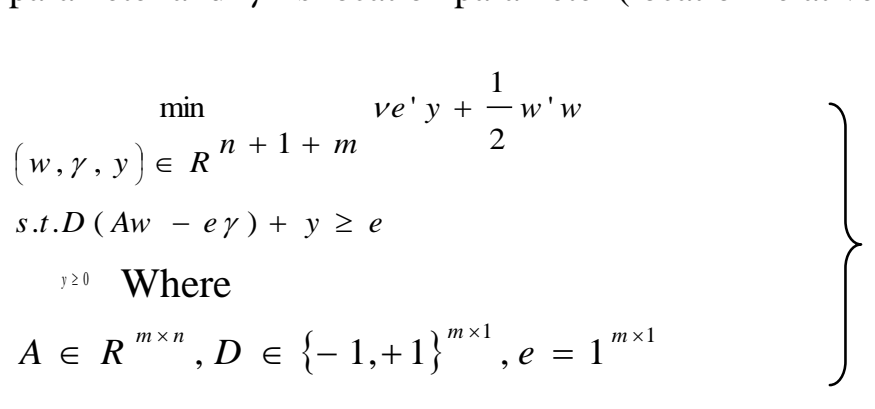

Vapnik [28] has shown that if the training features are separated without errors by an optimal hyper-plane, the expected error rate on a test sample is bounded by the ratio of the expectation of the support vectors to the number of training vectors. The smaller the size of the support vector set, more general the above result. Further, the generalization is independent of the dimension of the problem. In case such a hyper-plane is not possible, the 
next best is to minimize the number of misclassifications whilst maximizing the margin with respect to the correctly classified features.

\subsection{Classification using SVM}

For classification using SVM, two classes at a time are considered. There are four Hilbert transform features used as input and corresponding them there are four orientation parameters ' $w$ ', namely, $w_{1}, w_{2}$, and $w_{3}$. The adjusting parameter $\gamma$ are tabulated for different pairs of gear box conditions. From the Table 3 it can be observed that overall classification efficiency using SVM is $100 \%$.

For each condition feature vectors consisting of 100 feature value sets were collected from the experiment at different rpm. 70 samples in each class were used for training and 30 samples are reserved for testing SVM. Training was done and the values of ' $w$ ' (Weights) ' $\mathrm{g}$ ' (Gamma) and efficiency obtained for statistical features of Hilbert coefficients using SVM are tabulated Table 3.

The classification efficiency is of high value of $100 \%$ classification for all conditions of the gear box. The feature vectors corresponding to good gear were compared with those of the faulty gears at different loading and lubrication levels, by aking each fault at a time.

\section{Discussion}

1) The use of Hilbert transform and extraction of statistical features from Hilbert coefficients was found to be very efficient for classification using SVM.

2) Decision tree is a good tool in selecting the besfeatures among the extracted feature vectors. standard error, sample variance, standard deviation and median value were found to be the most contributing features.

3) In SVM, the parameters $w$ (Weight) and $\gamma$ (Gamma) define the separating plane. These can be used for testing a newset of data and classifying the faults accordingly. The efficiency for aripus conditions using SVM for Hilbert coefficients are shown in Table 3.

4) From Table 3 the classification capability of SVM is almost complete in all cases of gear box. It is able to identify the different faults, loading conditions and lubrication conditions correctly.

Table 3. Classification Efficiency of SVM for Hilbert Transform Coefficients

\begin{tabular}{|c|c|c|c|c|c|c|c|}
\hline SI No & Classification & Weight 1 & Weight 2 & Weight 3 & Weight 4 & Gamma & Efficiency $\%$ \\
\hline 1 & Good_Dry_No LoadV's GF_Dry_No Load & 1.006665 & 2.03453721 & -0.0564181 & -3.640595 & -0.3159363 & 100 \\
\hline 2 & Good_Dry_No Load Vs TCW_Dry_No Load & 2.007273 & 3.05116616 & 6.06362402 & 3.27170175 & -2.8774654 & 100 \\
\hline 3 & Good_Dry_No Load Vs TFW_Dry_No Load & 0.998164 & 1.96287944 & 6.73523696 & 6.36685875 & -3.5896555 & 100 \\
\hline 4 & Good_Dr_full Load Vs GTB_Dry_Full Load & 1.98888 & 0.78351989 & 11.3145288 & 7.52828921 & -5.2122483 & 100 \\
\hline 5 & Good_Dry Full/Load Vs TCW_Dry_Full Load & 2.098173 & 1.95187567 & -0.7138724 & -31.930282 & 4.70931127 & 100 \\
\hline 6 & Good_Dry_Full Load Vs TFW_Dry_Full Load & 0.982909 & 1.72108334 & 14.8951688 & 14.0782851 & -7.5201866 & 100 \\
\hline 7 & Good_Half Lub_No Load Vs GTB_Half Lub_No Load & -17.27814 & -409.19094 & -0.21145 & 18.1953954 & -6.3547209 & 100 \\
\hline 8 & Good_Half Lub_No Load Vs TCW_Half Lub_No Load & 2.078793 & 3.69586599 & 2.68880373 & -37.778647 & 3.11932479 & 100 \\
\hline 9 & Good_Half Lub_No Load Vs TFW_Half Lub_No Load & -2.718788 & -48.081575 & 86.5092587 & 393.365359 & -121.64171 & 100 \\
\hline 10 & Good_Half Lub_Full Load Vs GTB_Half Lub_Full Load & -6.1928 & -371.27767 & 0.27124712 & 0.78227149 & -2.9482374 & 100 \\
\hline 11 & Good_Half Lub_Full Load Vs TCW_Half Lub_Full Load & 1.095317 & \begin{tabular}{|l|l|}
1.8768679 \\
\end{tabular} & -0.4882311 & -32.326534 & 4.27775822 & 100 \\
\hline 12 & Good_Half Lub_Full Load Vs TFW_Half Lub_Full Load & -30.66933 & -754.89637 & 0.68438735 & 2.09541784 & -5.0627613 & 100 \\
\hline 13 & Good_Full Lub_No Load Vs GTB_Full Lub_No Load & -15.51331 & -436.52522 & -0.4765187 & -0.2177245 & -2.5928008 & 100 \\
\hline 14 & Good_Full Lub_No Load Vs TCW_Full Lub_No Load & 1.332811 & 3.9443439 & -15.229702 & -66.647869 & 15.6233331 & 100 \\
\hline 15 & Good_Full Lub_No Load Vs TFW_Full Lub_No Load & -47.01098 & -718.13552 & 1.66281572 & 3.4127699 & -5.5591077 & 100 \\
\hline 16 & Good_Full Lub_Full Load Vs GTB_Full Lub_Full Load & 0.807686 & -1.8920842 & 18.8713031 & 46.4293354 & -19.516966 & 100 \\
\hline 17 & Good_Full Lub_Full Load Vs TCW_Full Lub_Full Load & 9.856937 & 81.109495 & -12.584637 & -236.50271 & 40.3749388 & 100 \\
\hline 18 & Good_Full Lub_Full Load Vs TFW_Full Lub_Full Load & 0.69347 & -3.2274409 & 50.0446892 & 112.265759 & -47.962825 & 100 \\
\hline
\end{tabular}




\section{Conclusion}

Fault diagnosis of gear box is one of the core research areas in the field of condition monitoring of rotating machines. The method developed using Hilbert coefficients carry out the fault diagnosis of the gear box efficiently. The use of Hilbert transform is found to be extremely useful in fault diagnosis of rotating equipments like gear box. There was no misclassification and the classification efficiency is $100 \%$ for all the conditions of the gear box under investigation. The decision tree helps to filter and select unique features which help in condition monitoring of the gear box. Overall the paper demonstrates the power of Hilbert transform in condition monitoring of the gear box under investigation.

\section{References}

[1] G. Dalpiaz, A. Rivola and R. Rubini, "Effectiveness and sensitivity of vibration processing techniques for local fault detection in gears", Mechanical System and Signal Processing, vol. 14, no. 3,(2000), pp. 387412.

[2] I. Yesilurt, "Fault detection and location in gears by the smoothed instantaneous power spectrum distribution", NDT\&E Internation, vol. 36, (2003), pp. 535-542.

[3] G. Meltzer and N. Phong Dien, "Fault diagnosis in gears operating under non-stationary rotational speed using polar wavelet amplitude maps", Mechanical Systems and Signal Processing, vol. 18, no. 5, (2004), pp. 985-992.

[4] D. L. Jones and T. W. Parks, "A resolution comparison of several time-frequency representations", IEEE Transactions on Signal Processing, vol. 40, no. 2, (1992),pp. 413-420

[5] P. D. McFadden and W. J. Wang, "Analysis of gear vibration signatures by the weighted Wigner-Ville distribution", Vibrations in Rotating Machinery, Proceedings of the Institution of Mechanical Engineers, C432/134, (1992), pp. 393-397.

[6] J. Ma and C. J. Li, "G3ear defect detection through môdel-based wideband demodulation of vibrations", Mechanical Systems and Signal Processing, vol. 10, no. 5, (1996), pp. 653-665.

[7] W. J. Wang and P. D. McFadden, "Application of wavelets to gearbox vibration signals for fault detection", Journal of Sound and Vibration, vol. 192, no. 5, (1966),pp. 927-939.

[8] J. Lin and M. J. Zuo, "Gearbox fautt diagnosis using adaptive wavelet filter", Mechanical Systems and Signal Processing, vol. 17, no.6, (2003), pp. 1259-1269.

[9] W. J. Wang and P. D. Mckadden, "Application of orthogonal wavelet to early gear damage detection", Mechanical Systems and Signal Processing, vol. 9, no. 5, (1995), pp. 497-507.

[10] R. T. Ogden, "Essentian Wavelets for-Statistical Applications and Data Analysis", Birkhäuser, Boston, (1997), pp. 174-203.

[11] N. G. Nikøiaou and I. A. Antoniadis, "Rolling element bearing fault diagnosis using wavelet packet", NDT\&E International, vo1. 35.(2002), pp. 197-205.

[12] Y. Wu and R. Du, "Feature extraction and assessment using wavelet packets for monitoring of machining processes", Mechanica1 System and Signal Processing, vol. 10, no. 1, (1996), pp. 29-53.

[13] G. G. Yen and K. C. Lin, "Wavelet packet feature extraction for vibration monitoring", IEEE Transactions on Electronics, 47, no. 3, (2000), pp. 650-667.

[14] C. F. Bohren and D. R. Huffman, "Absorption and Scattering of Light by Small Particles", WileyInterscience, New York, (1983).

[15] T. B. Benjamin, "Internal waves of permanent form in fluids of great depth", J. FluidMech., vol. 25, (1967), pp. 559-592.

[16] H. Ono, Algebraic solitary waves in stratified fluids", J. Phys. Soc. Japan, vol. 39, (1975), pp. 1082-1091.

[17] N. E. Huang and Z. Shen, "The empirical mode decomposition and the Hilbert spectrum for nonlinear and non-stationary time series analysis", Proc. R. Soc. Lond.A, vol. 454, (1998), pp. 903-995.

[18] W. J. Wang and P. D. Mcfadden, "Mechanical Systems and Signal Processing", Early detection of gear failure by vibration analysis II: interpretation of the time-frequency distribution using image processing techniques, vol. 7, no. 3, (1983), pp. 205 -215.

[19] O. Petrille, B. Paya, I. I. Esat and M. N. M. Badi, "Proceedings of the Energy-Sources Technology Conference and Exhibition: Structural Dynamics and Vibration PD, vol. 70, (1995) 97.

[20] D. Boulahbal, M. F. Golnaraghi and F. Ismail, Proceedings of DETC'97, 1997 ASME Design Engineering Technical Conference, DETC97/VIB-4009, (1997). 
[21] F. A. Andrade, I. and M. N. M. Badi, "A new approach to time-domain vibration condition monitoring: Gear tooth fatigue crack detection and identification by the Kolmogrov-Smirnov test", Journal of Sound and vibration, vol. 240, no. 5, (2001), pp. 909-07.

[22] A. Carcaterra and A. Sestieri, "Complex envelope analysis: a quasi-static approach to vibrations", Journal of Sound and Vibration, vol. 201, no. 2, (1997), pp. 205-233.

[23] P. D. McFadden, "Detecting fatigue cracks in gears by amplitude and phase demodulation of the meshing vibration", ASME Transactions Journal of Vibration Acoustics Stress andReliability in Design, vol. 108, (1986), pp. 165-170.

[24] J. J. Zakrajsek, R. F. Handschuh, D. G. Lewicki and H. J. Decker, "Detecting gear tooth fracture in a high contact ratio face gear mesh", Proceedings of the 49th Meeting of the Mechanical Failure Prevention Group, Virginia Beach, VA, (1995) April 18-20.

[25] Y. H. Peng, P. A. Flach, P. Brazdil and C. Soares, "Decision tree-based data characterization for metalearning", ECML/PKDD-2002 Workshop IDDM-2002, Helsinki, Finland.

[26] V. Sugumaran, V. Muralidharan and K. I. Ramachandran, "Feature selection using decision tree and classification through Proximal Support Vector Machine for fault diagnostics of roller bearing", Mechanical Systems and Signal Processing, vol. 21, (2006), pp. 930-942.

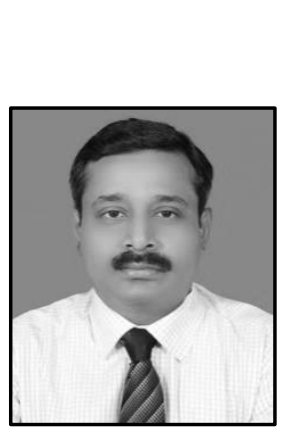

\section{Author}

Dr Saravanan Natarajan M.E.s PhD, the author has 15 years of experience of which 1 years are in teaching in engineering colleges in Sultanate of Omar and India at post graduate and undergraduate level. Is an active researcher in field of condition monitoring of mechanical equipment. Author also has 4 years industrial experience in the field of CAD/CAE. He has carried out many projects Or GEA, US Has worked in General Electric Appliances in Kourisville, Kentucky state, USA.

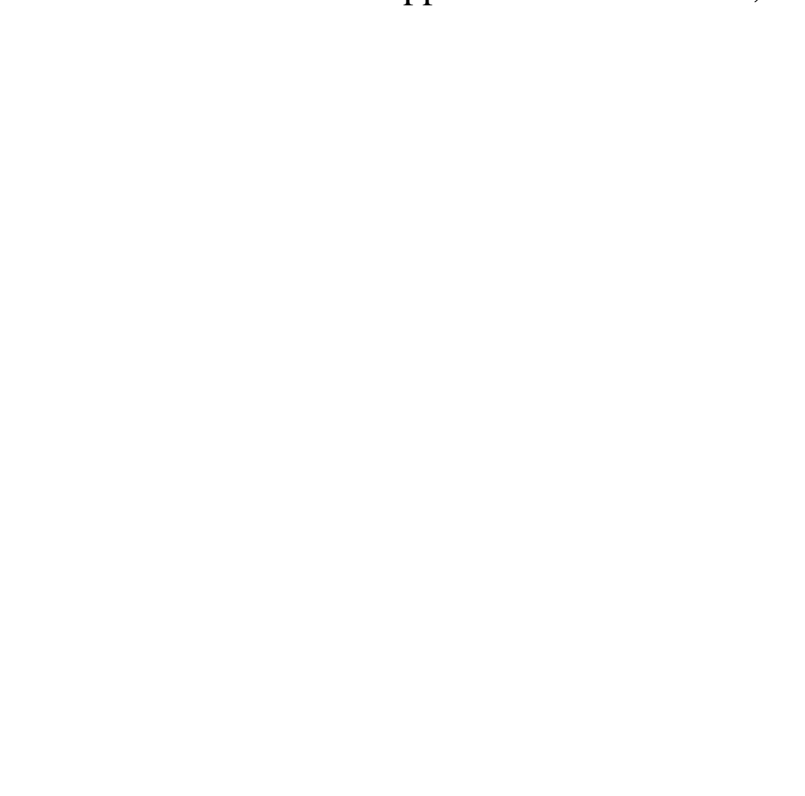

